\title{
A Versatile Fermentation Sampling Arrangement
}

\author{
BY N. G. HEATLEY \\ The Sir William Dunn School of Pathology, University of Oxford
}

SUMMARY: The sampling tube from the fermentation vessel is connected to a tube passing loosely through the specially fitted cap of a standard screw-capped bottle. By means of a mouthpiece suction can be applied so that a sample of fluid is drawn over into the bottle, which is then replaced by a similar empty sterile bottle. By fitting an extension of the tube to pass to the bottom of the bottle, and by blowing instead of sucking, fluid in the bottle may be driven over into the fermentation vessel. The device may be used for certain other operations such as removing or transferring sterile solutions or suspensions.

Among the desirable features of any arrangement for withdrawing samples from a pure culture fermentation are the following: (1) The risk of contamination of the main fermentation-and in some cases of the sample also-should be as small as possible. (2) There should be no dead space. If there is, the first part of the sample must be taken separately and rejected, or the sample must be large in relation to the volume of the dead space. (3) It should not be necessary to interrupt aeration or stirring of the fermentation. (4) The arrangement should not be unduly complicated or expensive.

It is believed that the device described below fulfils these requirements better than many of the sampling arrangements in common use. It has been useful for certain other aseptic operations.

\section{CONSTRUCTION}

This will be understood from the sectional drawing (1) in Fig. 1.

A screw-cap $a$, fitting a standard 1 or $4 \mathrm{oz}$. bottle $h$, is attached to a glass or metal tube $b$ of the same outside diameter by a rubber sleeve $c$. The top of the tube $b$ is closed by a rubber bung $d$ pierced with two holes. One of these carries a straight tube $e$ which passes loosely through a hole in the centre of cap $a$ and its washer. The lower end of tube $e$ is tapered slightly. The second hole in bung $d$ carries a short bent tube $f$ connected by rubber tubing to the mouthpiece g.

Tube $b$ is packed with cotton-wool by raising the bung slightly and feeding in the cotton-wool as a loose rope. As it is fed in, in a helical fashion, it is tamped down with a rod. Finally bung $d$ is pressed home and $e$ is adjusted so that its tip is $22 \mathrm{~mm}$. below the rubber washer in cap $a$. Once filled, the apparatus may be used again and again without repacking. It is, of course, sterilized with a bottle $h$ in position, as shown.

\section{METHODS OF USE}

As a fermentation sampler. The top end of tube $e$ is connected to a tube passing into the fermentation vessel and ending below the level of the fluid in it. (During the initial sterilization of the whole set-up it may be desirable to clamp 
off this connexion.) To take a sample, slight pressure is applied to the mouthpiece $g$ in order to drive out any medium from the dead space of the tube. Suction is then applied till the desired amount of medium has come over into bottle $h$. If the latter is below the level of the liquid in the fermenter or if the pressure in the latter is greater than atmospheric, it may be necessary to break

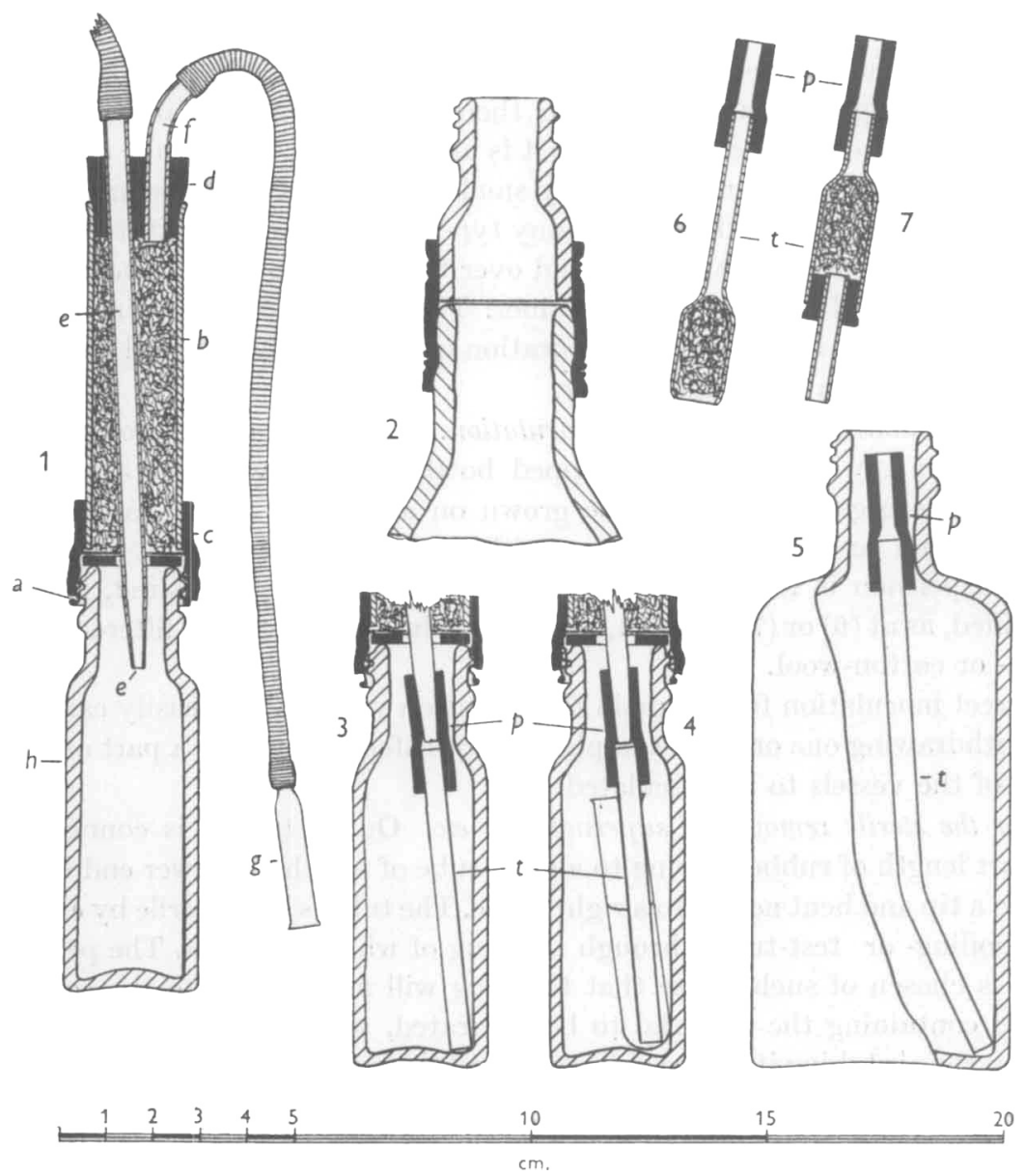

Fig. 1

the syphon by applying momentary pressure at $g$. A clean sterile bottle is now substituted for $h$, which in turn is closed with the cap from the clean bottle. This changing, which takes 4 or 5 sec., is the only time during which there is exposure to contamination.

The size of the sample may be varied from a few drops to a bottleful, and it is as easy to attach a $4 \mathrm{oz}$. as a $1 \mathrm{oz}$. bottle. Still larger samples may be taken with the device shown at (2) in the figure, consisting of the upper half of a $1 \mathrm{oz}$. screw-capped bottle attached to the neck of a larger bottle or flask by a rubber sleeve. 
For adding sterile solutions or inocula to a fermentation vessel. The solution to be added is made up and sterilized in the amount to be added in 1 or $4 \mathrm{oz}$. screwcapped bottles. The bottles contain, or have added to them, pieces of glass tubing $t$ the upper ends of which bear short pieces of pressure tubing $p$. If separately sterilized, for example in a plugged test-tube, the rubber should be at the bottom of the tube so that when tipped into the bottle of fluid to be added the rubber part is at the top. To transfer the fluid, the bottle containing it (and a tube $t p$ ) is substituted for $h$, the tapered tip of tube $e$ being guided into the pressure tubing $p$. Mouth pressure is then applied at $g$ until all the fluid has been driven over. For a $4 \mathrm{oz}$. bottle it is an advantage if the tube $t$ is slightly bent ((5) in Fig. 1). For adding very small amounts of fluid the arrangement shown at (4) may be used; but with any type of container quantitative transfer may be achieved by blowing the fluid over then sucking back some of the fermentation liquid to rinse the container. This procedure has been useful for making good the losses due to evaporation in simple small aerated cultures by the daily addition of distilled water.

For inoculation of the main fermentation. Cultures can be grown in fluid medium in any suitable screw-capped bottle then blown over as described above. The organism can also be grown on a slope or a thin layer of solid medium and suspended by shaking with water, with or without glass beads. The suspension is then blown over in the usual way. If desired, $t$ may be modified, as at (6) or (7) in Fig. 1, so that the fluid transferred is filtered through glass- or cotton-wool.

Direct inoculation from a main fermentation vessel is also easily carried out by withdrawing one or more samples and transferring each or a part of each to each of the vessels to be inoculated.

For the sterile removal of supernatants, etc. Outlet tube $e$ is connected by a short length of rubber tubing to a glass tube of which the lower end is drawn out to a tip and bent nearly to a right angle. The tube is kept sterile by enclosure in a boiling- or test-tube, through the plug of which it passes. The protecting tube is chosen of such a size that the plug will fit snugly into the centrifuge bottle containing the material to be separated, so that as the latter is withdrawn the air taking its place will be filtered through the plug. After the supernatant has been withdrawn the sediment may be washed with any desired liquid by attaching a bottle of it to screw-cap $a$ and blowing it over. The bent tip of the delivery tube enables a powerful swirling motion to be set up. If the sediment is not readily dispersed in the washing fluid this can be facilitated by sucking the suspension backwards and forwards several times. The supernatant or the resuspended sediment may be dispensed direct into a number of sterile bottles with the minimum chance of contamination. 